\title{
Methyl 3,4-Dihydroxybenzoate Enhances Resistance to Oxidative Stressors and Lifespan in C. elegans Partially via daf-2/daf-16
}

\author{
Xiang-Nan Mi ${ }^{1}$, Li-Fang Wang ${ }^{1,2}$, Yang $\mathrm{Hu}^{1}{ }^{\text {, Jun-Ping Pan }}{ }^{1}$, Yi-Rong Xin ${ }^{1}$, Jia-Hui Wang ${ }^{1}$, \\ Hai-Ju Geng ${ }^{1}$, Song-Hui Hu ${ }^{1}$, Qin Gao ${ }^{1}$ and Huan-Min Luo ${ }^{1,3, *}$ \\ 1 Department of Pharmacology, School of Medicine, Jinan University, Guangzhou 510632, China; \\ mrsmanjusaka@163.com (X.-N.M.); wlifang001@163.com (L.-F.W.); yanghu0325@163.com (Y.H.); \\ panjunpingjnu319@163.com (J.-P.P.); xinyirong@yeah.net (Y.-R.X.); wjh8623@126.com (J.-H.W.); \\ jiapin@outlook.com (H.-J.G.); jinanyaolihu326@163.com (S.-H.H.); tgaoq@jnu.edu.cn (Q.G.) \\ 2 School of Nursing, Guangdong Pharmaceutical University, Guangzhou 510632, China \\ 3 Institute of Brain Sciences, Jinan University, Guangzhou 510632, China \\ * Correspondence: tlhm@jnu.edu.cn; Tel.: +86-20-8522-8869
}

Received: 13 May 2018; Accepted: 31 May 2018; Published: 5 June 2018

\begin{abstract}
Genetic studies have elucidated mechanisms that regulate aging; however, there has been little progress in identifying drugs that retard ageing. Caenorhabditis elegans is among the classical model organisms in ageing research. Methyl 3,4-dihydroxybenzoate (MDHB) can prolong the life-span of $C$. elegans, but the underlying molecular mechanisms are not yet fully understood. Here, we report that MDHB prolongs the life-span of $C$. elegans and delays age-associated declines of physiological processes. Besides, MDHB can lengthen the life-span of eat-2 (ad1113) mutations, revealing that MDHB does not work via caloric restriction (CR). Surprisingly, the life-span-extending activity of MDHB is completely abolished in daf-2 (e1370) mutations, which suggests that daf-2 is crucial for a MDHB-induced pro-longevity effect in C. elegans. Moreover, MDHB enhances the nuclear localization of $d a f-16 / F o x O$, and then modulates the expressions of genes that positively correlate with defenses against stress and longevity in C. elegans. Therefore, our results indicate that MDHB at least partially acts as a modulator of the daf-2/daf-16 pathway to extend the lifespan of C. elegans, and MDHB might be a promising therapeutic agent for age-related diseases.
\end{abstract}

Keywords: Methyl 3,4-dihydroxybenzoate; ageing; Caenorhabditis elegans; daf-2; daf-16/FoxO; oxidative stress

\section{Introduction}

"Every man desires to live long but no man wishes to be old," Jonathan Swift said in the 17th century. Although treatments for aging would be desirable, the development of such treatments is challenging [1,2]. Approaches based on random screens of potential treatments require relevant and practicable assays of aging, but the time and effort needed to measure aging are substantial obstacles. To address these challenges, we exploited the C. elegans model system.

Caenorhabditis elegans (C. elegans) has been exploited as a classical model organism in aging research for its simple biology, self-fertilization, short lifespan, ease of culture, and ease of genetic analysis from the early 1970 s onward [3-6]. The observation that $C$. elegans has only $35 \%$ homology to human genes has long been seen as one of its chief deficits in anti-aging research [7,8]. Surprisingly, it has not prevented nematodes from becoming a classical model organism in biogerontological studies [8].

Methyl 3,4-dihydroxybenzoate (MDHB) has been reported to have antioxidant, neurotrophic, and neuroprotective effects [9-12]. We previously reported that MDHB might extend the adult 
lifespan of C. elegans partly through the W06A7.4 gene [13]. However, the exact mechanisms of the lifespan-extending activity of MDHB in C. elegans remain unclear. The studies described here were conducted to investigate the potential mechanisms of MDHB in lifespan extension.

Several genetic and environmental manipulations are associated with the life-span of C. elegans [14], such as the insulin/IGF-1 signaling (IIS) pathway, known as the most typical pathway regulating lifespan in many creatures. Confined IIS signaling regulates the insulin/IGF-1 transmembrane receptor (IGFR) ortholog daf-2; the core transcriptional factor forkhead box $\mathrm{O}(F o x O)$ transcription factor $d a f-16$ and its interaction with additional transcriptional factors in the nucleus could prolong the lifespan of nematodes and promote health in mammals [15-18]. In addition, the oxidative stress pathway that represents an imbalance between toxic reactive oxygen species (ROS) and antioxidant systems is considered to be connected to lifespan regulation in worms [19]. Furthermore, dietary restriction (DR) was proved to be the only intervention that successfully prolongs lifespan in mammals [20]. To investigate the relationships between MDHB and these regulators of aging, we exploited the C. elegans model system to investigate the effects and potential mechanisms of MDHB on the longevity of $C$. elegans.

The results suggest that MDHB significantly extends the lifespan of C. elegans without causing a reduction in production, and MDHB regulates the lifespan of worms by both a W06A7.4-dependent and W06A7.4-independent mechanism. Besides, MDHB inhibits the expression of daf-2/IIS, while it upregulates the expression of the daf-16 gene. Our genetic analysis shows that MDHB promotes daf-16/FoxO nuclear localization, thereby modulating the expression of daf-2/daf-16 target genes that are positively associated with stress tolerance and lifespan regulation, including the upregulation of genes encoding the antioxidant enzymes. Putting all this together, we conclude that MDHB acts as a modulator of the daf-2/daf-16 pathway to enhance resistance to oxidative stressors, ultimately prolonging the adult lifespan of $C$. elegans.

\section{Results}

\subsection{Exposure to MDHB during Adulthood Extends the Mean Lifespan of Wild-Type C. elegans}

In our previous study, worms cultured in an NGM (nematode growth media) medium containing $160 \mathrm{mg} / \mathrm{L}$ MDHB displayed the largest lifespan extension [13]. It was found that $160 \mathrm{mg} / \mathrm{L} \mathrm{MDHB}$ extended the mean adult lifespan of worms from $15.47 \pm 0.118$ days to $18.41 \pm 0.323$ days $(+19 \%$, $p<0.01)$, more than the positive drug resveratrol group $(+13.5 \%, p<0.05)$ (Figure $1 \mathrm{~A}$ and Table 1 ). A lifespan assay of worms pretreated with $160 \mathrm{mg} / \mathrm{L}$ MDHB from gestation to the fourth larval (L4) stage (MDHB 4/0) or from the L4 stage to death (MDHB 0/4) was conducted to investigate the action stage where MDHB worked to enhance lifespan. Exposure to MDHB from conception to fourth larval stage (MDHB 4/0) did not affect the lifespan of worms, while exposure to MDHB from L4 stage to death (MDHB 0/4) prolonged the adult lifespan to $18.41 \pm 0.323$ days $(+19 \%, p<0.01)$ (Figure 1B and Table 1). These findings show that MDHB functioned after the L4 stage to extend lifespan and had no effect on the developmental stage.

Table 1. Summary of mean life-spans of C. elegans.

\begin{tabular}{|c|c|c|c|c|c|c|c|c|}
\hline \multirow[b]{2}{*}{ Genotype } & \multirow[b]{2}{*}{ Treatment } & \multirow[b]{2}{*}{$\begin{array}{c}\text { Mean } \pm \\
\text { SEM (Days) }\end{array}$} & \multirow[b]{2}{*}{$\%$ Change } & \multirow[b]{2}{*}{ N (Censored) } & \multirow[b]{2}{*}{ EXP } & \multirow[b]{2}{*}{$p$} & \multicolumn{2}{|c|}{$95 \%$ CI for Mean } \\
\hline & & & & & & & $\begin{array}{l}\text { Lower } \\
\text { Bound }\end{array}$ & $\begin{array}{l}\text { Upper } \\
\text { Bound }\end{array}$ \\
\hline \multirow{5}{*}{$\mathrm{WT}, 20^{\circ} \mathrm{C}$} & None & $15.47 \pm 0.118$ & & $1132(168)$ & 13 & & 15.11 & 15.83 \\
\hline & $\operatorname{MDHB}(0 / 4) 160 \mathrm{mg} / \mathrm{L}$ & $18.41 \pm 0.323$ & +19 & $346(54)$ & 4 & 0.008 & 17.76 & 19.06 \\
\hline & $\operatorname{MDHB}(4 / 0) 160 \mathrm{mg} / \mathrm{L}$ & $15.02 \pm 0.235$ & -2.9 & $372(28)$ & 4 & 0.618 & 14.56 & 15.49 \\
\hline & RES $100 \mu \mathrm{M}$ & $17.56 \pm 0.316$ & +13.5 & $352(48)$ & 4 & 0.012 & 16.94 & 18.18 \\
\hline & RAPA $100 \mu \mathrm{M}$ & $18.76 \pm 0.332$ & +21.3 & $367(33)$ & 4 & 0.003 & 18.11 & 19.40 \\
\hline \multirow{2}{*}{ W101 } & None & $13.30 \pm 0.240$ & -16 & $512(88)$ & 6 & & 12.83 & 13.78 \\
\hline & MDHB & $14.84 \pm 0.276$ & +11.6 & 489 (111) & 6 & 0.018 & 14.30 & 15.38 \\
\hline \multirow{2}{*}{ W203 } & None & $13.01 \pm 0.260$ & -16 & $508(92)$ & 6 & & 12.50 & 13.52 \\
\hline & MDHB & $14.03 \pm 0.235$ & +7.9 & $492(108)$ & 6 & 0.042 & 13.57 & 14.52 \\
\hline
\end{tabular}


Table 1. Cont.

\begin{tabular}{|c|c|c|c|c|c|c|c|c|}
\hline \multirow[b]{2}{*}{ Genotype } & \multirow[b]{2}{*}{ Treatment } & \multirow[b]{2}{*}{$\begin{array}{c}\text { Mean } \pm \\
\text { SEM (Days) }\end{array}$} & \multirow[b]{2}{*}{$\%$ Change } & \multirow[b]{2}{*}{ N (Censored) } & \multirow[b]{2}{*}{ EXP } & \multirow[b]{2}{*}{$p$} & \multicolumn{2}{|c|}{$95 \%$ CI for Mean } \\
\hline & & & & & & & $\begin{array}{l}\text { Lower } \\
\text { Bound }\end{array}$ & $\begin{array}{l}\text { Upper } \\
\text { Bound }\end{array}$ \\
\hline \multirow{2}{*}{ W213 } & None & $13.78 \pm 0.270$ & -11 & $522(78)$ & 6 & & 13.25 & 14.32 \\
\hline & MDHB & $15.01 \pm 0.281$ & +9 & $501(99)$ & 6 & 0.038 & 14.46 & 15.56 \\
\hline \multirow{2}{*}{ daf-16 (mu86) } & None & $13.15 \pm 0.337$ & -15 & $546(54)$ & 6 & & 12.55 & 13.75 \\
\hline & MDHB & $14.07 \pm 0.306$ & +7 & $523(77)$ & 6 & 0.036 & 13.41 & 14.72 \\
\hline \multirow{2}{*}{ daf-2 (e1370) } & None & $29.24 \pm 0.469$ & +89 & $532(68)$ & 6 & & 28.32 & 30.16 \\
\hline & MDHB & $30.06 \pm 0.459$ & +2.8 & $513(87)$ & 6 & 0.512 & 29.16 & 30.96 \\
\hline \multirow{2}{*}{ eat-2 (ad1113) } & None & $18.77 \pm 0.265$ & +21.3 & $576(24)$ & 6 & & 18.25 & 19.29 \\
\hline & MDHB & $22.60 \pm 0.316$ & +20.4 & $543(57)$ & 6 & 0.039 & 21.98 & 23.22 \\
\hline
\end{tabular}

All strains were fed live E. coli OP50 and cultured at $20^{\circ} \mathrm{C}$. The concentrations were represented in milligrams per liter for methyl 3,4-dihydroxybenzoate (MDHB). Genotypes with no drug treatment are compared with line 1. Otherwise, comparisons are to the same genotype with no drug treatment. For these comparisons in the columns showing life-spans that were shown as Mean \pm S.E.M (days) in column 3. Column 2, worms with or without drug treatment. $\mathrm{N}$, the total number of worms were tested. EXP, the number of independent experiments.
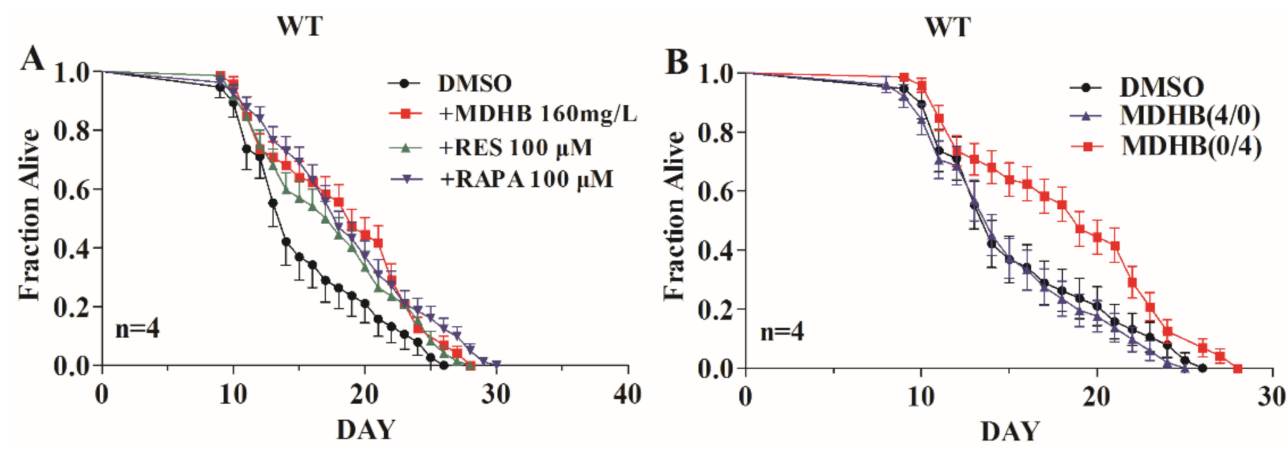

Figure 1. Methyl 3,4-dihydroxybenzoate (MDHB) extends adult life span of wild-type nematodes. (A) Survivorship curve of wild-type (WT) nematodes under different treatments. $100 \mu \mathrm{M}$ resveratrol (+RES) and $100 \mu \mathrm{M}$ rapamycin (+RAPA) were taken as the positive controls, separately. It was found that treatment with MDHB (Methyl 3,4-dihydroxybenzoate) $(160 \mathrm{mg} / \mathrm{L})$, resveratrol $(100 \mu \mathrm{M})$ and rapamycin $(100 \mu \mathrm{M})$ could significantly $(p<0.05)$ extend the mean lifespan of animals compared to the Normal control group. (B) Worms were exposed to MDHB (160 mg/L) from conception to L4 (+MDHB 4/0), or to MDHB from L4 to death (+MDHB 0/4). External drug concentrations are shown in milligrams per liter for MDHB. All data are described as mean \pm S.E.M, the statistical details are summarized in Table 1.

\subsection{MDHB Reduces Lipofuscin Accumulation}

Animals exposed to $160 \mathrm{mg} / \mathrm{L}$ MDHB had a reduced accumulation of lipofuscin, known as a biomarker for cellular damage and an autofluorescent protein that is cumulative in the progress of senescence $[21,22]$. Compared to untreated controls, intestinal lipofuscin levels at day 10 were reduced by $23.2 \%$ in MDHB-treated animals $(p<0.01)$ and $20 \%$ in resveratrol-treated groups $(p<0.01)$ (Figure 2A,B). Generally, lifespan extension of nematodes was related to decreased lipofuscin. Consequently, MDHB-induced reduction of lipofuscin demonstrated that MDHB could decrease oxidative protein damage, ultimately leading to a decelerated aging process in nematodes. 

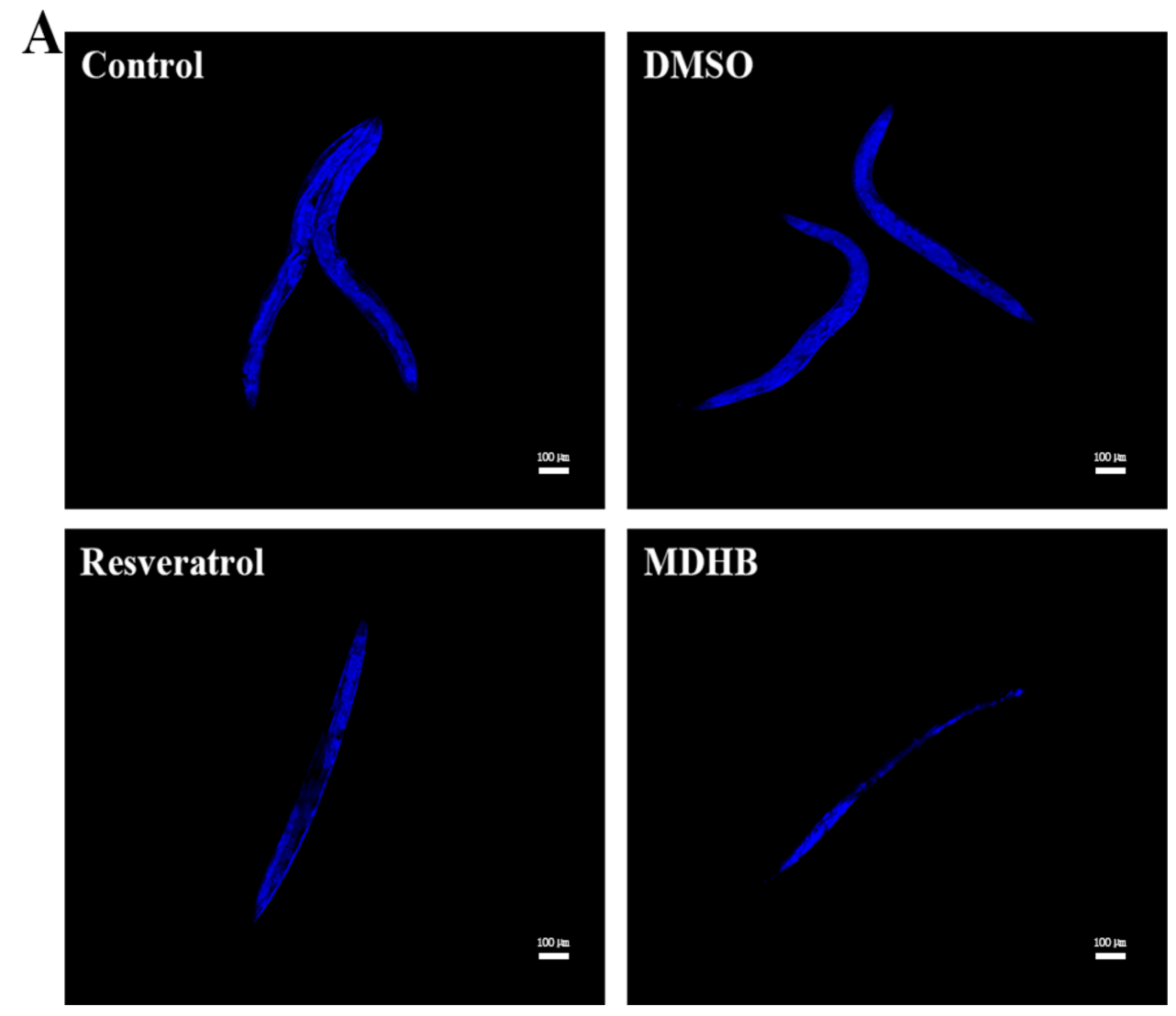

\section{MDHB}

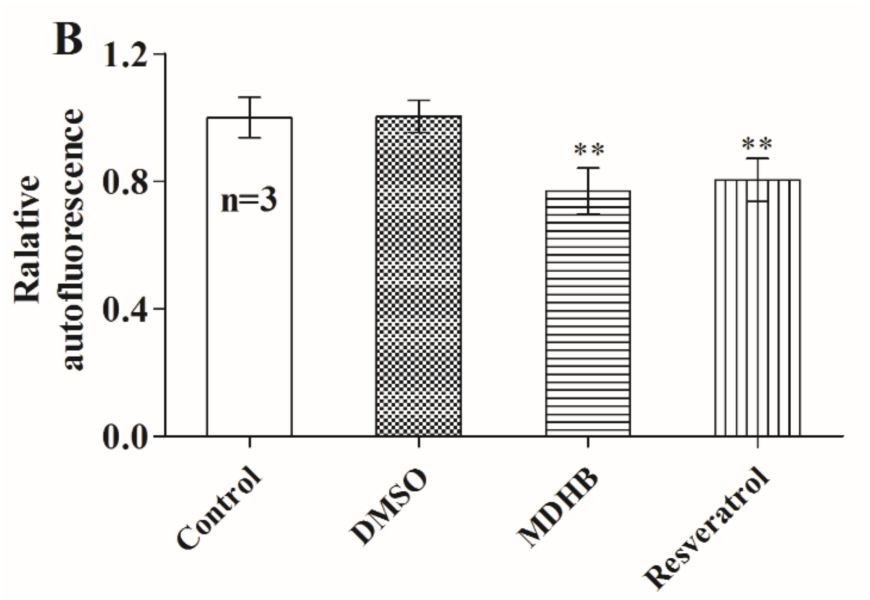

Figure 2. Effect of MDHB on lipofuscin accumulation of wild-type C. elegans. (A) Typical fluorescence images of worms in untreated control, Dimethyl sulfoxide (DMSO)-treated, resveratrol-treated and MDHB-treated worms are shown. (B) Effect of MDHB on intracellular lipofuscin accumulation in wild-type C. elegans at day 10. Fluorescence intensities of the lipofuscin fluorescence of 30 worms from different groups were analyzed by the ImageJ soft. Data are shown as means \pm S.E.M comparing to the untreated control (OD-background $/ \mathrm{mm}^{2}=465.32 \pm 31.56$; set to 1 ). Statistical significance of differences between treated and control groups were described significant at $* * 0.01$ by one-way ANOVA with LSD post hoc test. ANOVA, analysis of variance. This experiment was performed three times.

\subsection{Effects of $M D H B$ on $S O D$ Activity in C. elegans}

MDHB is known to protect wild-type animals against thermal pressure [13]. Superoxide dismutase (SOD), an important antioxidant enzyme in organisms, plays a vital role in the elimination of some harmful substances produced in the metabolic process, like reactive oxygen species (ROS). In this study, 
we found that worms pretreated with $160 \mathrm{mg} / \mathrm{L}$ MDHB showed higher SOD activity compared to the DMSO (dimethyl sulfoxide) treated groups (Figure 3). That is to say, the antioxidant activity of MDHB could be a substantial contributor to its lifespan-extending activity in worms. Conducting further experiments on the effects of antioxidant MDHB in higher organisms would be indispensable in the late stage of this research.

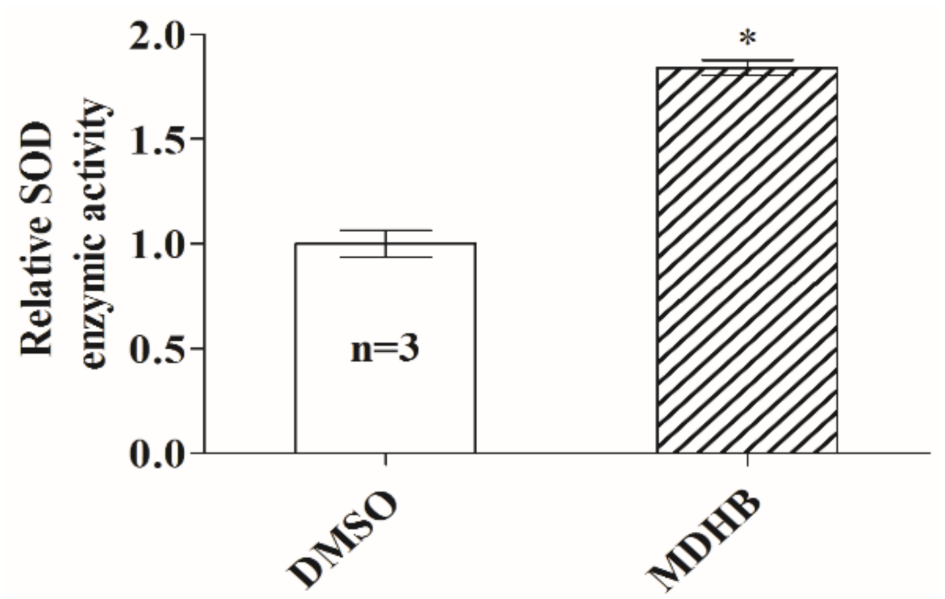

Figure 3. Effect of MDHB on superoxide dismutase (SOD) activity of wild-type C. elegans. Error bars represent the standard deviations (SD), and differences between the DMSO (control) and MDHB groups were described significant at $*<0.05$ by one-way ANOVA. This experiment was performed thrice.

\subsection{MDHB Extends the Lifespan of W06A7.4 Loss-of-Function Mutants}

MDHB might extend the lifespan of wild-type C. elegans via the W06A7.4 gene [13]. Therefore, three W06A7.4 gene loss-of-function strains (W101, W203, and W213) were used to investigate the relationship between the W06A7.4 gene and the pro-longevity action of MDHB in worms. It was found that wild-type worms (15.47 \pm 0.118 days) lived longer than W101 strains (13.30 \pm 0.240 days, $p<0.05), W 203$ strains $(13.01 \pm 0.260$ days, $p<0.05)$, and W213 strains $(13.78 \pm 0.270$ days, $p<0.05)$ (Figure 4A and Table 1). However, our results show that exposure to MDHB extended the lifespan of all three W06A7.4 loss-of-function mutants (W101, W203, and W213 strains) (Figure 4B-D and Table 1), but the percentage change caused by MDHB was less than that in wild-type worms (Figure 4E and Table 1). Thus, we report that the longevity action of MDHB may partly require W06A7.4, and partial MDHB-induced longevity is independent of the W06A7.4 gene in worms.
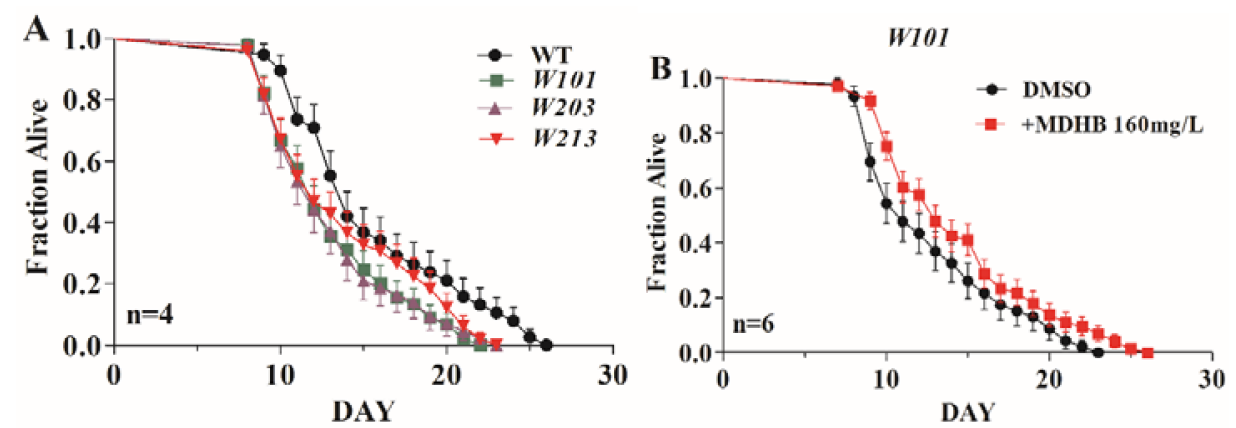

Figure 4. Cont. 

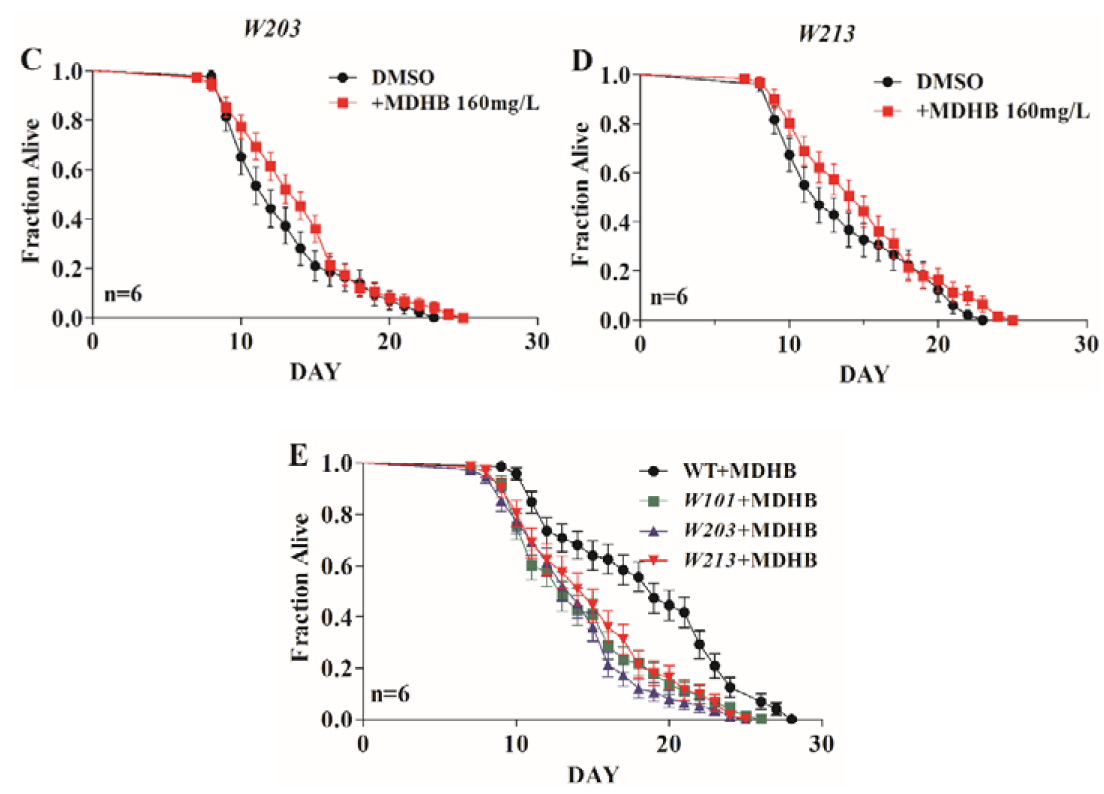

Figure 4. Effects of MDHB on the mean lifespan of W06A7.4 loss-of-function mutations (W101, W203, and W213 strains). (A) Survival of the three W06A7.4 mutations (W101, W203, and W213) compared to wild-type animals. The mutated worms (W101, W203, and W213) had shorter lifespans than the wild-type N2 worms, $p<0.05$. (B-D) Survival of mutated worms with W06A7.4 gene knocked out, with and without MDHB treatment. Treatment with MDHB notably enhanced mean lifespan of three W06A7.4 loss-of-function mutants, W101 (+11.6\%, $p<0.05)$, W203 $(+7.9 \%, p<0.05)$, and W213 $(+9 \%, p<0.05)$. (E) Survival of three W06A7.4 loss-of-function mutations (W101, W203, and W213 strains) and wild-type animals exposed to MDHB. Data are represented as mean \pm S.E.M; statistical details are summarized in Table 1 .

\subsection{MDHB Does Not Appear to Lengthen Lifespan via Caloric Restriction}

Lifespan is modulated by various genetic and environmental elements; among them, caloric restriction is recognized as an irrefutable environmental modulator of lifespan in many species, including C. elegans [23-26]. Here, there was no significant difference in fat storage, food intake, and body patterning, because worms treated with MDHB did not become thin or starved. Furthermore, MDHB could extend the lifespan of wild-type worms without the reduced fecundity that usually occurs with nutrient limitation [13]. Combining the results, MDHB remarkably prolonged the mean lifespan of eat-2 (ad1113) mutations, a genetic model of dietary restriction (DR), from $18.77 \pm 0.265$ days to $22.60 \pm 0.316$ days $(+20.4 \%, p=0.039)$ (Figure 5 and Table 1$)$. Above all, we report that MDHB does not appear to enhance lifespan via caloric restriction.

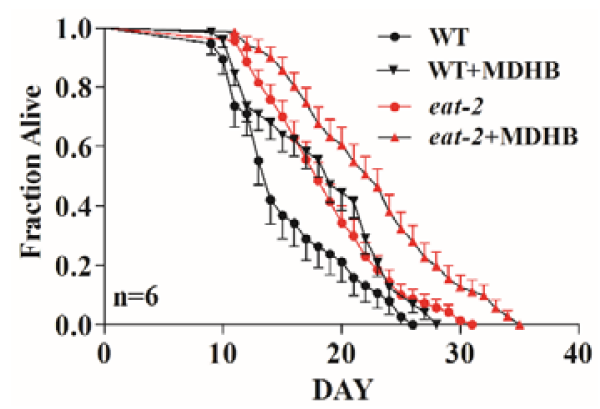

Figure 5. Effects of MDHB on the lifespan of eat-2 (ad1113) mutants. MDHB notably prolonged lifespan of eat-2 (ad1113) mutations from $18.77 \pm 0.265$ days to $22.60 \pm 0.316$ days $(+20.4 \%, p=0.039)$. Each experiment was repeated at least six times. The data are represented as mean \pm S.E.M, the statistical details are generalized in Table 1. 


\subsection{MDHB Prolongs the Lifespan of C. elegans Partially through the daf-2/daf-16 Signaling Pathway}

The insulin/insulin-like growth factor (IIS) signaling pathway plays a vital role in lifespan regulation and other important biological processes in C. elegans and other creatures. The IIS pathway is modulated by insulin-like peptide ligands that bind to the insulin/IGF-1 transmembrane receptor (IGFR) ortholog daf-2. daf-2/IGFR regulates the activity of a conserved phosphoinositide 3-kinase (PI3K)/Akt kinase cascade, ultimately leading to the modulation of a forkhead family transcription factor, daf-16, that controls most of the functions of the IIS pathway [27-29]. Here, we found that MDHB enhanced the longevity of the daf-16 loss-of-function mutations $(+7 \%, p<0.05)$ (Figure 6A and Table 1$)$, but less than the percentage change in wild-type animals $(+19 \%, p<0.01)$ (Figure $6 \mathrm{~B}$ and Table 1$)$, indicating that part of the MDHB-induced lifespan extension is independent of daf-16 and part of the action may demand daf-16. Moreover, there was no obvious difference in the mean lifespan between daf-2 (e1370) mutations exposed to $160 \mathrm{mg} / \mathrm{L}$ MDHB or not (Figure 6C and Table 1). Furthermore, we found that worms with MDHB treatment showed a significantly lower expression of daf-2, but a higher expression of daf-16 (Figure 6D). Taken together, these findings indicate that MDHB may prolong the lifespan of nematodes partially through the daf-2/daf-16 signaling pathway.
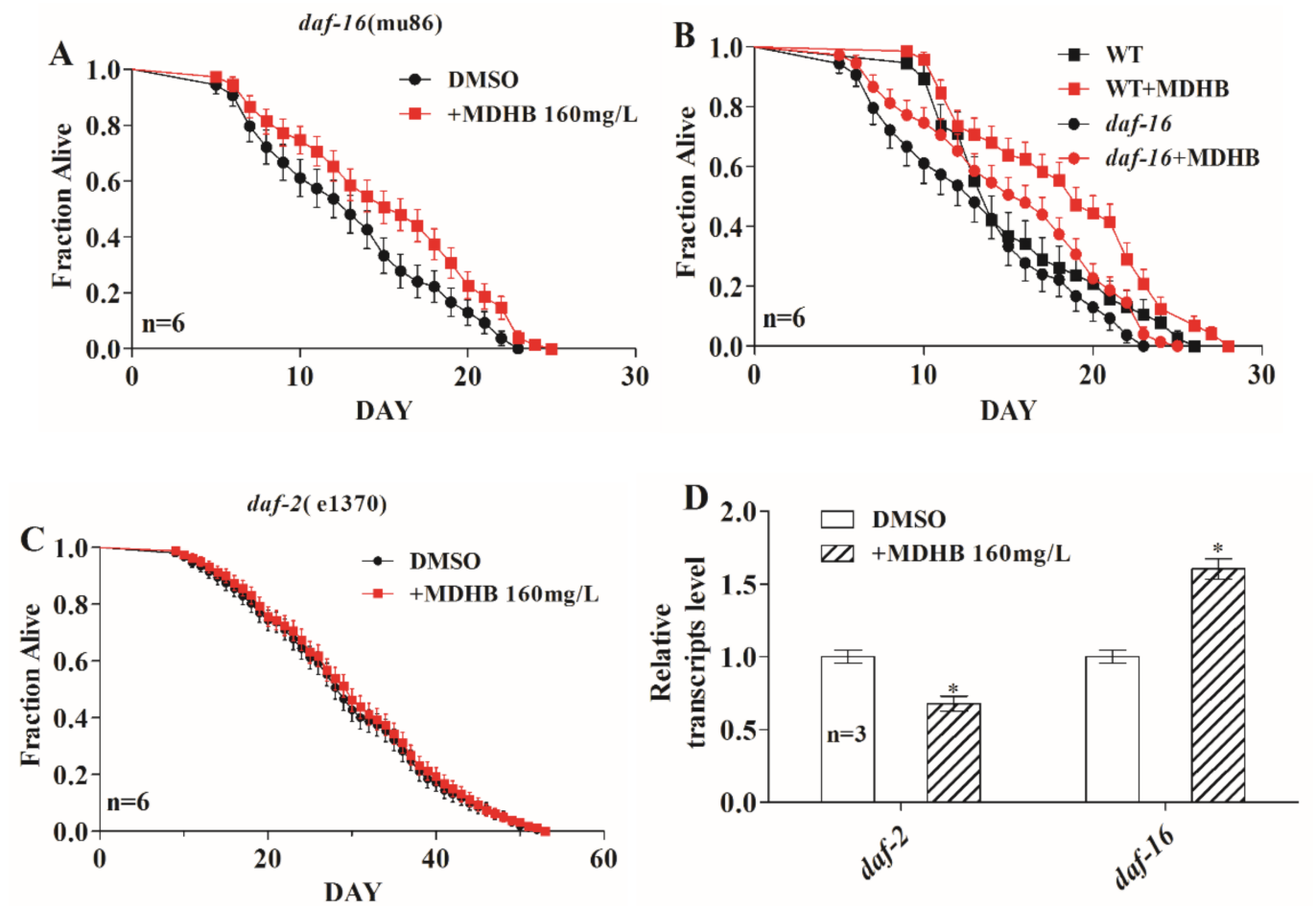

Figure 6. Effects of MDHB on the lifespan of daf-2 and daf-16 loss-of-function mutants. (A) Effects of MDHB on the lifespan of daf-16 (mu86) mutations. (B) Effects of MDHB on the lifespan of Wild-Type (WT) animals and daf-16 (mu86) mutants. (C) on the daf-2 (e1370) mutants. (D) Quantification of MDHB exposure on the expression levels of $d a f-2$ and $d a f-16$ by RT-PCR (real-time polymerase Chain Reaction) in wild-type nematodes. Error bars represent the standard deviations (SD), and differences between the DMSO (control) and MDHB groups were described significant at $*<0.05$ by one-way ANOVA. actin-1 was used as an endogenous control, and the mRNA expression were determined by real-time PCR using the $2^{-\Delta \Delta C t}$ method, ${ }^{*} p<0.05$.

\subsection{MDHB Promotes daf-16/FoxO Nuclear Localization}

Suppressing the daf-2 signaling pathway transferred daf-16 from cytoplasm into nucleus, where it modulates the expression of genes that are known to be associated with longevity regulation and diverse cellular stress responses [16]. We visualized the effect of MDHB on daf-16 intracellular location 
through a specific transgenic strain (TJ356) that included the daf-16::GFP (green fluorescent protein) reporter construct integrated in the genome. Exposure to $160 \mathrm{mg} / \mathrm{L}$ MDHB induced $11.5 \%$ nuclear accumulation of daf-16::GFP in nuclei, but only $2.4 \%$ in worms in the untreated control groups (Figure 7), suggesting that MDHB might act upstream of daf-16 and enhance its nuclear translocation. These results strongly indicate that daf-16 is an indispensable part of the action of MDHB-induced promotion of antioxidant ability in nematodes, similar to other phenolic antioxidants.
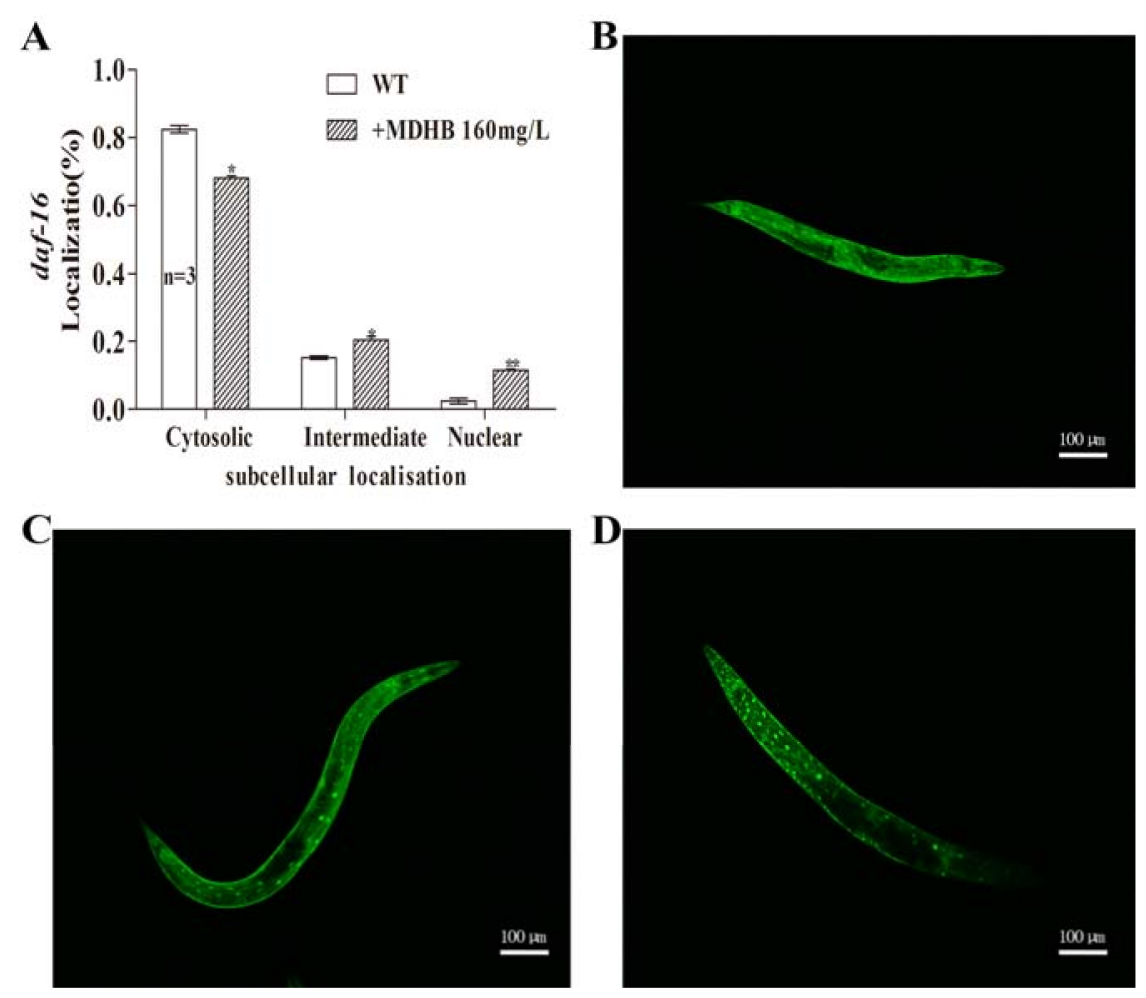

Figure 7. MDHB induced nuclear localization of daf-16. (A) Data from different group exposure to $160 \mathrm{mg} / \mathrm{L}$ MDHB or not are described as means \pm S.E.M to show the percentile of animals displaying cytosolic, intermediate or nuclear of localization. Statistical significance among animals of the control groups and MDHB groups was analyzed by one-way ANOVA followed by Bonferroni (post-hoc) correction $\left({ }^{*} p<0.05, * * p<0.01\right)$. (B-D) Micrographs elucidate typical daf-16 localization in TJ356 transgenic strains. (B) daf-16 mainly locating in the cytoplasm, (C) mainly locating in cytoplasm and nucleus, (D) mainly in the nucleus. This experiment was repeated thrice.

\subsection{Effects of $M D H B$ on the Expression of daf-2/daf-16 Target Genes}

In C. elegans, many genes and pathways are associated with oxidative stress response and longevity regulation (e.g., IIS signaling pathway, CR pathway). Previous research reported that an increase in oxidative stress tolerance was associated with prolonged lifespan in nematodes $[19,30,31]$. The result that MDHB protected wild-type worms from oxidative damage while it did not enhance stress resistance in daf-16 (mu86) mutants (Figure 8A) suggested that daf-16 is essential for the observed MDHB-mediated lifespan enhancement under oxidative stress resistance. Strains CF-1553 and CL-2070 were used to examine the transcriptional levels of sod-3 and $h s p-16.2$ that are positively related to lifespan and oxidative stress [31,32]. Here, the expression of GFP fused hsp-16.2 or sod-3 was significantly up to $47 \pm 5.75 \%$ and $76 \pm 7.05 \%$ with MDHB treatment (Figure $8 \mathrm{~B}, p<0.001$ ). We report that MDHB promotes daf-16 entering into the nucleus and enhances the transcriptional levels of sod-3 and $h s p-16.2$ to protect worms from oxidative stress. Above all, these results put forward a supposed mechanism of action of MDHB in C. elegans (Figure 8C). 
To further investigate whether the improved oxidative stress resistance of C. elegans pretreated with MDHB depends on daf-16-regulated activity, a microarray profiling analysis was used to examine the effects of MDHB on the following daf-2/daf-16 targeted genes: skn-1 [33,34], cst-1 [35], dod-22 [36], $h s p-12.1, h s p-16.1, h s p-16.49$ [36], mtl-1 [36], ins-18 [36], and sodh-1 [37]. The profiles of the differentially expressed genes (MDHB treatment vs. DMSO treatment, fold change $>2$ or $<0.5$ ) were obtained by analyzing the MDHB-regulated genes on the 6th day respectively within the three batches of the samples. The details about these genes are summarized in Table 2. 8 genes were confirmed by matching differentially expressed genes on the 6th day (Figure 8D). The corresponding functional annotations of these genes are shown in Table 2. Expression of $s k n-1, h s p-12.1, h s p-16.1, h s p-16.49, m t l-1$ and sodh-1 was significantly increased (fold change $>2, p<0.05$ ), while dod-22 and mtl-2 significantly decreased (fold change $>2$ or $<0.5, p<0.05$ ) (Figure 8D and Table 2). Together, these results suggest that MDHB prolongs the lifespan of $C$. elegans at least partially through the daf-2/daf-16 pathway, which is connected to oxidative stress resistance and longevity regulation.

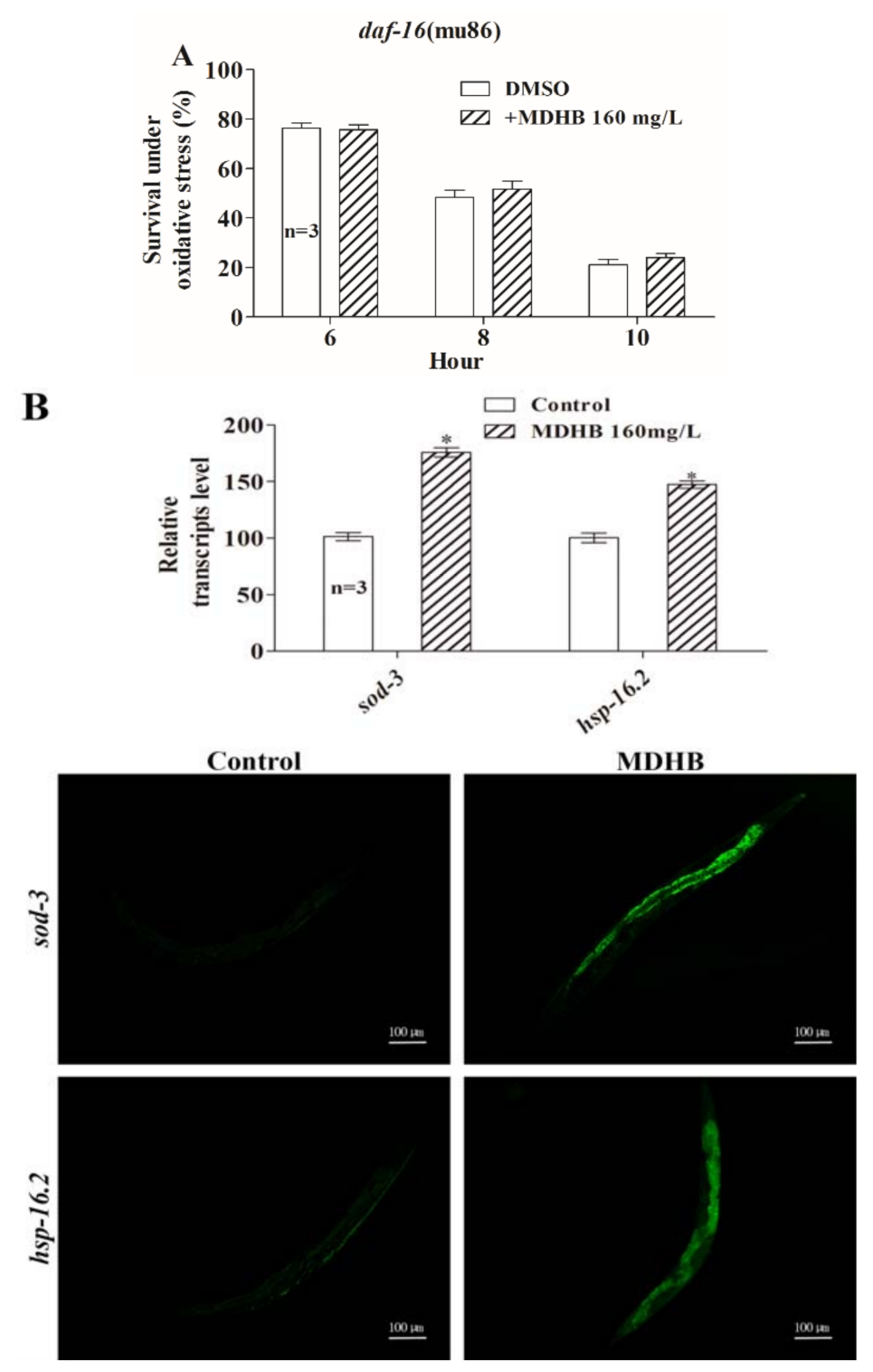

Figure 8. Cont. 

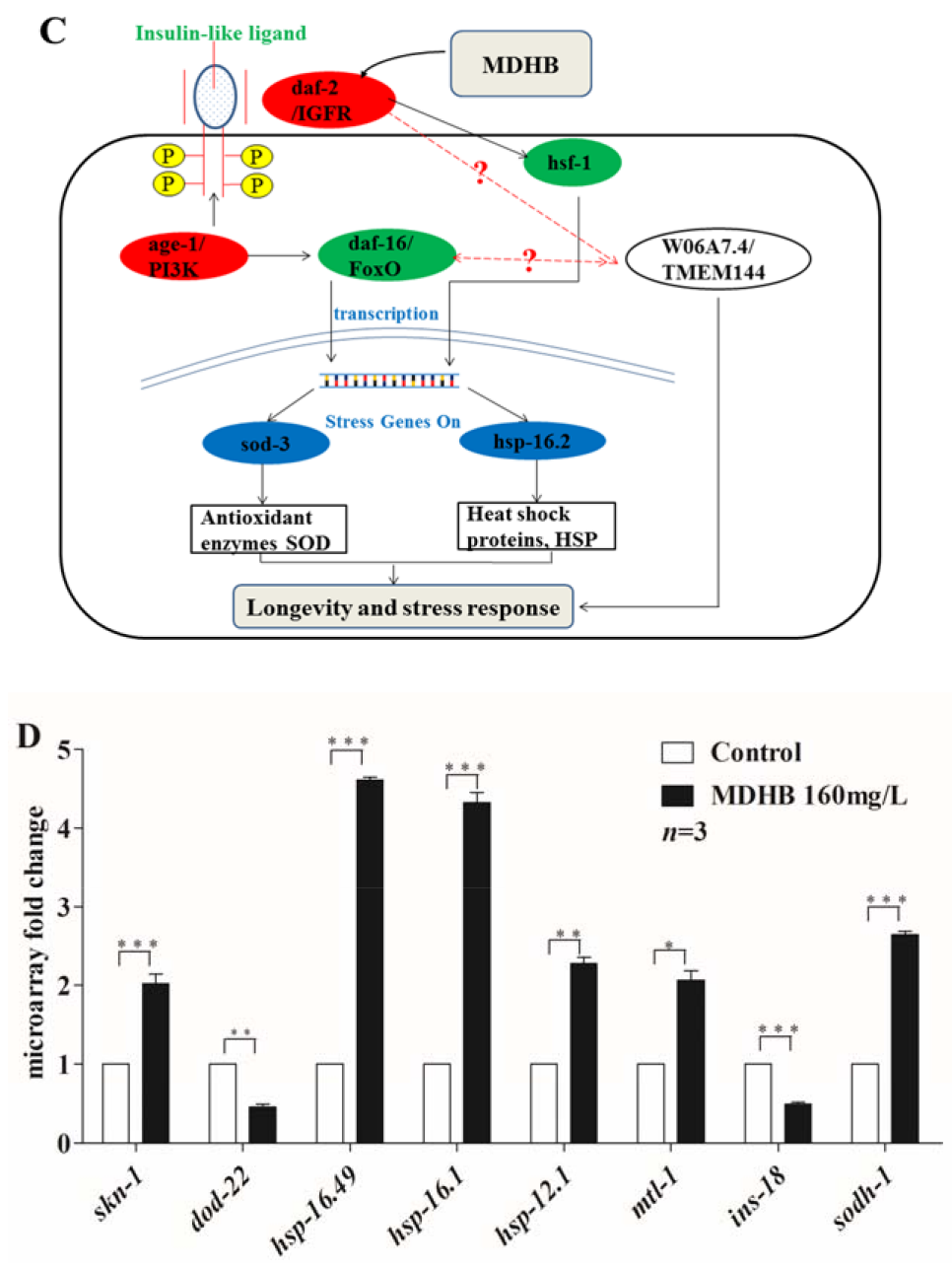

Figure 8. Influence of MDHB on the expression of daf-2/daf-16 target genes. (A) Influence of MDHB on survival rates of daf-16 (mu86) mutants under oxidative stress conditions induced by $\mathrm{H}_{2} \mathrm{O}_{2}$. MDHB did not enhance stress resistance in daf-16 (mu86) mutants. (B) Influence of MDHB on the expression of $h s p-16.2$ and sod-3 genes under normal conditions. Green fluorescent protein (GFP) was tested and quantified using a fluorescence multi-reader, and analyzed by the standard Student $t$-test. (C) A drawing of daf-2/daf-16 signaling and its mammalian homologues shows the supposed mechanism of action of MDHB in C. elegans. Active IIS promotes the phosphorylation-dependent cytoplasmic sequestration of the transcription factors daf-16/FoxO and $h s f-1$. The insulin/IGF-1 receptor ortholog daf-2 and other pathway components that promote IIS are colored red, and molecules that either antagonize IIS or are antagonized by IIS are colored green. Genes that are promoted by daf-16/FoxO are colored blue. Molecular, which may either promote or antagonize the activity of its target genes, are marked with black arrows. Genes that either antagonize daf-2/daf-16 or are antagonized by daf-2/daf-16 are marked with red arrows. Abbreviations: IIS, insulin/insulin-like growth factor-1 signaling; IGFR, Insulin-like growth factor 1 receptor; PI3K, phosphoinositide 3-kinase; FoxO, forxhead box protein O; P, the phosphate groups. (D) Influence of MDHB on the expression of partial daf-2/daf-16 pathway targets. The effects of MDHB on the expression of genes were detected through a microarray profiling analysis. ${ }^{*} p<0.05 ;{ }^{* *} p<0.01 ;{ }^{* * *} p<0.001$; ns: not significant. 
Table 2. Effects of MDHB on the expression of partial daf-2/daf-16-dependent targets.

\begin{tabular}{|c|c|c|c|c|}
\hline \multirow{2}{*}{ Cosmid No. } & \multirow{2}{*}{ Gene Symbol } & \multirow{2}{*}{ Brief Description } & \multicolumn{2}{|c|}{ MDHB Treatment } \\
\hline & & & Fold Change & $p$ Value \\
\hline T19E7.2 & skn-1 & $\begin{array}{l}\text { orthologous to the mammalian Nrf (Nuclear factor-erythroid-related } \\
\text { factor) transcription factors }\end{array}$ & 2.01 & $<0.001$ \\
\hline F55G11.5 & dod-22 & $\begin{array}{l}\text { DUF141 domain of unknown function, high similarity to } \\
\text { uncharacterized C. elegans K10D11.2 }\end{array}$ & -2.22 & 0.0023 \\
\hline T22A3.2 & $h s p-12.1$ & a member of the small heat shock family of proteins & 2.27 & 0.0045 \\
\hline T27E4.8 & $h s p-16.1$ & Member of the C. elegans hsp-16 family & 4.32 & $<0.001$ \\
\hline T27E4.3 & $h s p-16.49$ & Hsp20/alpha crystallin family, similar to alpha-B crystalline & 4.61 & $<0.001$ \\
\hline K11G9.6 & $m t l-1$ & Metallothionein-related cadmium-binding protein & 2.06 & 0.0083 \\
\hline T28B8.2 & ins-18 & $\begin{array}{l}\text { Insulin-like protein of the type- } \beta \text { subfamily; may be a ligand for the } \\
\text { daf- } 2 \text { receptor }\end{array}$ & -2.04 & 0.0421 \\
\hline K12G11.3 & sodh-1 & Alcohol dehydrogenase 1 , have oxidoreductase activity & 2.64 & $<0.001$ \\
\hline
\end{tabular}

\section{Discussion}

We previously reported that MDHB has antioxidant, neurotrophic, and neuroprotective effects [9-12]. Besides, MDHB extends the lifespan of C. elegans [13]. However, the mechanism by which MDHB extends lifespan remains hard to determine. The studies described here were aimed at exploring the exact mechanisms of lifespan-extending activity of MDHB in C. elegans.

First, our results demonstrate that MDHB prolongs C. elegans lifespan and delays the age-related declines of physiological processes. Moreover, we observe that worms exposed to MDHB show higher SOD activity and reduced accumulation of intracellular lipofuscin, which are associated with longevity regulation in worms. These findings raise a crucial question: what is the exact mechanism of lifespan-extending action of MDHB in C. elegans?

As previously reported, a variety of elements have been demonstrated to prolong the lifespan of worms, and the studies described here have clarified the relationship between MDHB and these elements.

Among the numerous factors that regulate lifespan, caloric restriction (CR) is recognized as an indisputable environmental modulator of lifespan in many species, including C. elegans [23,24]. $\mathrm{CR}$ prolongs lifespan and can be caused by a mutation of the eat-2 gene, which plays a vital role in pharyngeal pumping $[25,26]$. MDHB obviously prolonged the lifespan of eat-2 (ad1113) mutations (20.4\%) (Figure 5 and Table 1), suggesting that the primary mechanism of MDHB-induced longevity in worms is not caloric restriction. Besides, wild-type worms pretreated with MDHB were not nutrient-limited, as the animals did not display obvious differences in reproduction, fat storage, or somatotype (showing thin or starved) that always occur with CR [26].

As previously reported, the mechanism of MDHB in lifespan extension might be associated with the W06A7.4 gene in worms. Here, we report that three W06A7.4 loss-of-function mutations (W101, W203, W213) had shorter lifespans than wild-type N2 worms. However, the lifespan of three W06A7.4 mutations could be further extended by MDHB, but the percentage change caused by MDHB in W06A7.4 mutations was lower than that in wild-type nematodes. One explanation is that the reduced effect of MDHB is in agreement with other probabilities, including the detrimental consequences of pleiotropy caused by combining a mutation with MDHB. In addition, these results indicate that part of the longevity action of MDHB may require W06A7.4, and part of MDHB-induced lifespan-extending activity is independent of the W06A7.4 gene.

The insulin/IGF signaling pathway is a classical pathway that governs compressive capacity, dauer formation, and longevity [14,27]. Loss-of-function daf-16 mutations reduce lifespan and suppress lifespan extensions caused by mutations in upstream signaling pathway genes such as daf-2. Worms with MDHB treatment showed a significantly lower expression of daf-2, but a higher expression of daf-16 (Figure 6D). We also observed that MDHB exposure significantly prolonged the lifespan of daf-16 (mu86) mutants (7\%), though less than the percentage change in wild-type animals (19\%), indicating that MDHB regulates the lifespan of worms by both daf-16-dependent and 
daf-16-independent mechanisms. However, treatment with MDHB did not further extend the lifespan of daf-2 (e1370) mutants with defects in daf-2, revealing that daf-2 is not related to the mechanism of MDHB-induced long life in C. elegans.

We previously found that part of the lifespan-extending activity of MDHB in worms may need W06A7.4 and daf-16. Combining the result that daf-2 is not connected to the longevity action of MDHB in worms, these results suggest that MDHB at least partly works as a daf-2/daf-16 modulator to extend lifespan in C. elegans. Furthermore, this paper puts forth the idea that the W06A7.4 gene may function downstream of daf-16 or in parallel to daf-16/FoxO, but in the daf-2-mediated IIS pathway to regulate the adult lifespan of C. elegans. That is to say, the relationship between W06A7.4 and daf-2/daf-16 remains to be seen in further experiments (Figure 8C).

Aging has been related to oxidative stress resistance, and various studies have proved that lifespan modulation affects resistance to oxidative stress [19]. Accordingly, we performed an oxidative stress resistance assay to analyze the effects of MDHB on oxidative stress, and our studies showed that MDHB protected wild-type animals against oxidative damage, while it did not extend mean lifespan of daf-16 (mu86) loss-of-function mutations $(p>0.05)$ under oxidative stress. This finding indicates that daf-16 leads to the protective effect of MDHB in worms. Moreover, worms pretreated with MDHB showed enhanced daf-16 nuclear translocation and promoted expression of GFP guided by $h s p-16.2$ or sod-3, which functions downstream of daf-16. sod-3 is a typical scavenger enzyme of ROS in oxidative stress, and it catalyzes the disproportionation of active superoxide anions to molecular oxygen. One explanation is that the actions of MDHB are modulated, at least partially, by activating $h s p-16.2$ and sod-3 genes that are positively related to lifespan regulation and stress resistance. We suggest that a readjustment of MDHB on daf-16 leads to the verified antioxidant effects of MDHB in C. elegans.

To further investigate whether the improved resistance to oxidative stress of C. elegans pretreated with MDHB depends on daf-2/daf-16-regulated activity, a microarray profiling analysis was conducted to examine the effects of MDHB on the following daf-2/daf-16 target genes that are involved in oxidative stress response and ageing [34]. It was found that the expression of $s k n-1, h s p-12.1, h s p-16.1$, $h s p-16.49, m t l-1$, and sodh-1 was significantly increased, while dod-22 significantly decreased in worms exposed to MDHB, indicating that daf- 16 is necessary for the antioxidant effect of MDHB in C. elegans. Earlier research indicated that daf-16 functioned with other genes or transcription factors to regulate stress resistance and longevity in C. elegans [36]. In C. elegans, skn-1 is homologous to mammalian nuclear factor erythroid-related factor (Nrf) transcription factors. skn-1 functions in parallel to daf-16 to regulate stress resistance and aging in worms [33,34]. ins-18, a daf-2 antagonist, functions downstream of daf-16 to regulate the lifespan of C. elegans [36]. Lifespan extension was also found in ins-18 loss-of-function mutations [36]. Under conditions of stress or reduced daf-2 signaling, daf-16 entered into the nucleus, then modulated the transcription levels of genes associated with oxidative stress response and aging. Furthermore, MDHB exposure upregulated the expression of genes encoding antioxidant enzymes such as superoxide dismutase (sod-3) [36] metallothionein ( $m+1-1)$ [36], and sorbitol dehydrogenase (sodh-1) [37], which were predicted to have oxidoreductase activity [36]. Loss of function dod-22 increases the lifespan of worms [36]. MDHB downregulated the expression of dod-22 in worms. In addition, daf-16 functions with $h s f-1$ to regulate the expression of genes encoding the small heat-shock proteins. Upregulation of $h s p-16.1, h s p-16.49$, and $h s p-12.1$ were found in worms with MDHB treatment.

Taken together, these results suggest that MDHB enhances resistance to oxidative stressors and increases lifespan in nematodes at least partially by modulating daf-2/daf-16. However, more experiments must be conducted to investigate the underlying mechanisms of MDHB induced lifespan-extending action as the molecular targets of MDHB are not yet fully understood. 


\section{Materials and Methods}

\subsection{Chemicals}

MDHB (Tokyo Chemical Industry, Tokyo, Japan) was dissolved in DMSO (Sigma-Aldrich, St. Louis, MO, USA) at a concentration of $160 \mathrm{mg} / \mathrm{mL}$ and conserved it at $4^{\circ} \mathrm{C}$. The purity of MDHB was confirmed to be more than $98 \%$.

\subsection{General Methods and Strains}

N2 (wild-type), eat-2 (ad1113), daf-16 (mu86), daf-2 (e1370), TJ356 (zIs356 IV), CF1553 (muIs84), CL-2070 (dvIs70) strains were used in this study. All nematodes were cultivated at $20{ }^{\circ} \mathrm{C}$ on culture dishes $(60 \mathrm{~mm})$ containing nematode growth medium and a thick lawn of Escherichia coli OP50 bacteria [5]. N2 strain and streptomycin-resistant E. coli OP50 were amicably provided by Xiao-Chen Wang (National Institute of Biological Sciences, Beijing, China). Other strains were kindly provided by from Pei Zhong (School of Pharmaceutical Science, Sun Yat-sen University, Guangzhou, China). Coeval nematodes gained by treatment with alkaline hypochlorite [38].

\subsection{W06A7.4 Gene Knockout Through a CRISPR-Cas9 System}

Earlier experiments confirmed that the W06A7.4 gene might be associated with lifespan-extending activity of MDHB in C. elegans. Three W06A7.4 gene loss-of-function mutations were gained from the wild-type worms by a CRISPR-Cas9 gene editing system, including two mutant strains (W203, W213), in which two transcripts, W06A7.4b and W06A7.4a, were disrupted, and another mutant (W101) had only a transcript W06A7.4a mutation [5,38-43].

\subsection{Lifespan Assay}

Animals were cultivated at $20^{\circ} \mathrm{C}$ on culture dishes $(60 \mathrm{~mm})$ containing nematode growth medium with drugs or not and a thick lawn of Escherichia coli OP50. To obtain coeval nematodes, 10 20 pregnant nematodes were picked up to a fresh culture dish in the absence or presence of $160 \mathrm{mg} / \mathrm{L} \mathrm{MDHB}$ for egg-laying about 4-6 h, or treated with alkaline hypochlorite [44]. Synchronous L4 larvae were defined as day 0 and observed every day until death. Animals were transferred to fresh plates every day till the end of propagative period, and then transferred to new dishes about every 4 days thereafter. Animals displaying no autonomic movement or response when touched were mounted as dead. Censored data containing dead worms that procreated progeny internally, and manifested an extruded gonad or dehydration owing to wall climbing [3].

\subsection{Lipofuscin Accumulation Assay}

Age-synchronous L4 larvae were cultured in the presence of 5-Fluorouracil $(100 \mu \mathrm{M})$ and maintained with or without $160 \mathrm{mg} / \mathrm{L}$ MDHB for 10 days for intracellular lipofuscin accumulation test. Ten day old wild-type nematodes were pipetted on a microscope slide with $2 \%$ agar pad and anesthetized with levamisole ( $5 \mathrm{mM}, 3 \mathrm{~min}$ ). An Olympus X71 fluorescent microscope (Olympus X71, Olympus, Tokyo, Japan) was applied to capture the autofluorescence of lipofuscin [45]. The experiment has been repeated thrice. The standard Student's $t$-test was employed for statistical analysis.

\subsection{Measurement of SOD Activity}

To assess superoxide dismutase (SOD) activity, wild-type worms were cultured in NGM plates containing $160 \mathrm{mg} / \mathrm{L} \mathrm{MDHB}$ or not for 6 days. Animals on the sixth day of adulthood were collected from different plates with M9 buffer and washed thrice. Afterward, the harvested animals were suspended in M9 buffer and homogenized on ice [46]. The EnSpire Multimode Plate Reader (PerkinElmer, Waltham, MA, USA) was used to evaluate SOD activity according to an SOD assay kit (WST-1 method) (Nanjing Jiancheng, Nanjing, China). 


\subsection{Oxidative Stress Resistance Assay}

Oxidative stress resistance of worms was analyzed according to the method described formerly with a bit of modification. Briefly, hydrogen peroxide $\left(\mathrm{H}_{2} \mathrm{O}_{2}\right)$ induced oxidative stress assays were performed at $20^{\circ} \mathrm{C}$ as for lifespan assays, except hydrogen peroxide was added to NGM medium at final concentration of $3 \mathrm{mM}$. Synchronous L4 larvae were cultured under different conditions (control and $160 \mathrm{mg} / \mathrm{L}$ MDHB groups) for $72 \mathrm{~h}$ at $20^{\circ} \mathrm{C}$. The 3-day Adult hermaphrodites were incubated at $20^{\circ} \mathrm{C}$ on NGM plates containing $3 \mathrm{mM} \mathrm{H}_{2} \mathrm{O}_{2}$, an intracellular free-radical-generating compound, and a thick lawn of Escherichia coli OP50 to prevent nematodes from starvation response. Afterwards the survival ship of worms were recorded every hour until all nematodes died [47]. The dead worms were counted as described in the method of lifespan assay. Briefly, survivals of worms was monitored by touch-provoked movement method [48]. The experiment was repeated thrice, independently.

\subsection{Expression of sod-3 and hsp-16.2}

Age-synchronized CF1553 worms steadily expressing a sod-3::GFP fusion protein and CL2070 worms steadily expressing a $h s p-16.2::$ GFP fusion protein were treated with $160 \mathrm{mg} / \mathrm{L}$ MDHB under normal condition at $20^{\circ} \mathrm{C}$ for $6 \mathrm{~d}$. Worms were placed on the microscope slide coated with $2 \%$ agar anaesthetised with levamisole $(5 \mathrm{mM}, 3 \mathrm{~min})$. GFP were monitored with a confocal microscope (Olympus X71, Olympus, Tokyo, Japan). For quantitative analysis of GFP expression, 20 worms were randomly selected from both experimental groups and placed into a 96-well plate (a single worm in each well). The GFP fluorescence intensity of each worm from different groups was monitored with a confocal microscope (Zeiss, Göttingen, Germany) and quantified with a fluorescence multi-reader (PerkinElmer, USA; $n=20$ ) [49]. This experiment was repeated three times. The standard Student's $t$-test was employed for statistical analysis.

\section{9. daf-16 Localization}

Age-synchronized strain TJ356 worms were treated with $160 \mathrm{mg} / \mathrm{L} \mathrm{MDHB}$ at $20^{\circ} \mathrm{C}$ for 6 days. Then, day- 6 TJ356 nematodes were mounted on the glass slides coated with $2 \%$ agar anaesthetised with levamisole ( $5 \mathrm{mM}, 3 \mathrm{~min})$, and daf-16 location of worms were determined through a fluorescence microscope (Zeiss, Göttingen, Germany) at 100× magnification. daf-16 localization of each worm were classified into 3 types (cytosolic, intermediate and nuclear) in consideration of the main location of daf-16::GFP [49]. Intracellular location of the daf-16::GFP of each worm was analysed using the Image software (NIH, Bethesda, MA, USA).

\subsection{Microarray Profiling Analysis}

Age-synchronized wild-type L4 larvae were cultured under different conditions (untreated control, $1 \%$ DMSO, and $160 \mathrm{mg} / \mathrm{L}$ MDHB groups) in the presence of 5 -fluorouracil $(100 \mu \mathrm{M})$ at $20^{\circ} \mathrm{C}$ for 6 days. Then, day- 6 adult worms of different groups were collected for microarray profiling analysis (Shanghai Biotechnology Corporation, Shanghai, China). Total RNA was extracted using TRIZOL reagent (Cat\#15596-018, Life Technologies, Carlsbad, CA, USA) following the manufacturer's instructions and checked for a RIN (RNA INtegrity) number to inspect RNA integrity by an Agilent Bioanalyzer 2100 (Agilent Technologies, Santa Clara, CA, USA). Qualified total RNA was further purified by RNeasy Micro Kit (Cat \#74004, Qiagen GmBH, Hilden, Germany) and RNase-Free DNase Set (Cat \#79254, Qiagen) and then used for microarray analysis.

\section{Conclusions}

Here, we show that a readjustment of MDHB on daf-2/daf-16 leads to verified lifespan-extending action of MDHB in C. elegans. MDHB inhibits the expression of daf-2/IIS, while it upregulates the expression of the daf-16 gene. Then, MDHB promotes daf-16/FoxO nuclear localization, 
thereby modulating the expression levels of its target genes that are positively associated with stress tolerance and lifespan regulation, including the upregulation of genes encoding the antioxidant enzymes $h s p-16.2, h s p-16.49, h s p-16.41, m t l-1$, and sod-3. Given the evidence, we conclude that MDHB acts as a modulator of the daf-2/daf-16 pathway to enhance resistance to oxidative stressors, ultimately prolonging the adult lifespan of C. elegans.

Author Contributions: X.-N.M. performed experiments, analyzed data, and wrote the manuscript. L.-F.W. assisted in performing the experiments and data analyses. Y.H. assisted in analyzing the data and writing the manuscript. Q.G., J.-P.P. and Y.-R.X. contributed reagents/materials/analysis tools. J.-H.W. performed antibacterial assays. H.-J.G., S.-H.H. assisted in data analyses. H.-M.L. led this study, conceived and designed the experiments.

Funding: This research was funded by the National Natural Science Foundation of China (Grant No. 81473296).

Acknowledgments: We thank Xiao-Chen Wang and Pei Zhong for providing some worm strains, Shanghai Biomodel Organism Science \& Technology Development Co. Ltd. for CRISPR-Cas9 analysis, and Shanghai Biotechnology Corporation for microarray profiling analysis. Bristol N2 and E. coli OP 50 were provided by Xiaochen Wang (National Institute of Biological Sciences, Beijing, China). Other strains were a kind gift from Pei Zhong (School of Pharmaceutical Science, SUN Yat-sen University, Guangzhou).

Conflicts of Interest: The authors declare no conflict of interest.

\section{Abbreviations}

MDHB Methyl 3,4-dihydroxybenzoate

C. elegans Caenorhabditis elegans

IIS Insulin/Insulin-like growth factor-1 signaling pathway

daf-16 Orthology of the FoxO in C. elegans

FoxO Forxhead box protein $\mathrm{O}$

$\mathrm{CR} \quad$ Calorie restriction

DMSO Dimethyl sulfoxide

ROS Reactive oxygen species

SOD Superoxide Dismutase

RES Resveratrol

RAPA Rapamycin

5-FuDR 5-fluorouracil deoxyribotside

E. coli Escherichia coli

NGM Nematode growth medium

LB Luria-Bertani

RIN RNA integrity

\section{References}

1. Honda, Y.; Honda, S. Life span extensions associated with upregulation of gene expression of antioxidant enzymes in Caenorhabditis elegans; studies of mutation in the age-1, PI3 kinase homologue and exposure to hyperoxia. J. Am. Aging Assoc. 2002, 25, 21-28. [CrossRef] [PubMed]

2. Boccardi, V.; Mecocci, P. Telomerase activation and human health-span: An open issue. Aging Clin. Exp. Res. 2017. [CrossRef] [PubMed]

3. Evason, K.; Huang, C.; Yamben, I.; Covey, D.F.; Kornfeld, K. Anticonvulsant Medications Extend Worm Life-Span. Science 2005, 307, 258-262. [CrossRef] [PubMed]

4. Regitz, C.; Fitzenberger, E.; Mahn, F.L.; Dußling, L.M.; Wenzel, U. Resveratrol reduces amyloid- $\beta$ $\left(\mathrm{A} \beta_{1-42}\right)$-induced paralysis through targeting proteostasis in an Alzheimer model of Caenorhabditiselegans. Eur. J. Nutr. 2016, 55, 741-747. [CrossRef] [PubMed]

5. Brenner, S. The Genetics of Caenorhabditis elegans. Genetics 1974, 77, 71-94. [PubMed]

6. Sulston, J.E.; Brenner, S. The DNA of Caenorhabditis elegans. Genetics 1974, 77, 95-104. [PubMed]

7. Johnson, T.E.; Wood, W.B. Genetic analysis of life-span in Caenorhabditis elegans. Proc. Natl. Acad. Sci. USA 1982, 97, 6603-6607. [CrossRef] 
8. Tissenbaum, H.A. Using C. elegans for aging research. Invertebr. Reprod. Dev. 2015, 59 (Suppl. 1), $59-63$. [CrossRef] [PubMed]

9. Cai, L.; Wang, L.F.; Pan, J.P.; Mi, X.N.; Zhang, Z.; Geng, H.J.; Wang, J.H.; Hu, S.H.; Zhang, W.; Gao, Q.; et al. Neuroprotective effects of methyl 3,4-Dihydroxybenzoate against TBHP-induced oxidative damage in SH-SY5Y cells. Molecules 2016, 21, 1071. [CrossRef] [PubMed]

10. Zhou, X.; Su, C.F.; Zhang, Z.; Wang, C.Y.; Luo, J.Q.; Zhou, X.W.; Cai, L.; Yan, L.; Zhang, W.; Luo, H.M. Neuroprotective Effects of Methyl 3,4-dihydroxybenzoate Against $\mathrm{H}_{2} \mathrm{O}_{2}$-Induced Apoptosis in RGC-5 Cells. J. Pharmacol. Sci. 2014, 125, 51-58. [CrossRef] [PubMed]

11. Zhang, Z.; Zhou, X.; Zhou, X.; Xu, X.; Liao, M.; Yan, L.; Lv, R.; Luo, H. Methyl 3,4-dihydroxybenzoate promotes neurite outgrowth of cortical neurons cultured in vitro. Neural Regen. Res. 2012, 7, 971-977. [PubMed]

12. Zhang, Z.; Cai, L.; Zhou, X.; Su, C.; Xiao, F.; Gao, Q.; Luo, H. Methyl 3,4-dihydroxybenzoate promote rat cortical neurons survival and neurite outgrowth through the adenosine A2a receptor/PI3K/Akt signaling pathway. Neuroreport 2015, 26, 367-373. [CrossRef] [PubMed]

13. Zhang, W.; Cai, L.; Geng, H.J.; Su, C.F.; Yan, L.; Wang, J.H.; Gao, Q.; Luo, H.M. Methyl 3,4-dihydroxybenzoate extends the lifespan of Caenorhabditis elegans, partly via W06A7.4 gene. Exp. Gerontol. 2014, 60, 108-116. [CrossRef] [PubMed]

14. Murphy, C.T.; Hu, P.J. Insulin/insulin-like growth factor signaling in C. elegans. WormBook 2013, 1-43. [CrossRef] [PubMed]

15. Yen, K.; Mobbs, C.V. Evidence for only two independent pathways for decreasing senescence in Caenorhabditis elegans. AGE. 2010, 32, 39-49. [CrossRef] [PubMed]

16. Henderson, S.T.; Johnson, T.E. daf-16 integrates developmental and environmental inputs to mediate aging in the nematode Caenorhabditis elegans. Brief Commun. 2001, 11, 1975-1980. [CrossRef]

17. Grala, T.M.; Kay, J.K.; Walker, C.G.; Sheahan, A.J.; Littlejohn, M.D.; Lucy, M.C.; Roche, J.R. Expression analysis of key somatotropic axis and liporegulatory genes in ghreline and obestatin-infused dairy cows. Domest. Anim. Endocrinol. 2010, 39, 76-83. [CrossRef] [PubMed]

18. Kenyon, C. The plasticity of aging: Insights from long-lived mutants. Cell 2005, 120, 449-460. [CrossRef] [PubMed]

19. Baumeister, R.; Schaffitzel, E.; Hertweck, M. Endocrine signaling in Caenorhabditis elegans controls stress response and longevity. J. Endocrinol. 2006, 190, 191-202. [CrossRef] [PubMed]

20. Lakowski, B.; Hekimi, S. The genetics of caloric restriction in Caenorhabditis elegans. Proc. Natl. Acad. Sci. USA 1998, 95, 13091-13096. [CrossRef] [PubMed]

21. Brunk, U.T.; Terman, A. Lipofuscin: Mechanisms of age-related accumulation and influence on cell function. Free Radic. Biol. Med. 2002, 33, 611-619. [CrossRef]

22. Berdichevsky, A.; Nedelcu, S.; Boulias, K.; Bishop, N.A.; Guarente, L.; Horvitz, H.R. 3-Ketoacyl thiolase delays aging of Caenorhabditis elegans and is required for lifespan extension mediated by sir-2.1. Proc. Natl. Acad. Sci. USA 2010, 107, 18927-18932. [CrossRef] [PubMed]

23. Lee, G.D.; Wilson, M.A.; Zhu, M.; Wolkow, C.A.; de Cabo, R.; Ingram, D.K.; Zou, S. Dietary deprivation extends lifespan in Caenorhabditis elegans. Aging Cell 2006, 5, 515-524. [CrossRef] [PubMed]

24. Mair, W.; Dillin, A. Aging and survival: The genetics of life span extension by dietary restriction. Annu. Rev. Biochem. 2008, 77, 727-754. [CrossRef] [PubMed]

25. Kaeberlein, T.L.; Smith, E.D.; Tsuchiya, M.; Welton, K.L.; Thomas, J.H.; Fields, S.; Kennedy, B.K.; Kaeberlein, M. Lifespan extension in Caenorhabditis elegans by complete removal of food. Aging Cell 2006, 5, 487-494. [CrossRef] [PubMed]

26. Bishop, N.A.; Guarente, L. Two neurons mediate diet-restriction-induced longevity in C. elegans. Nature 2007, 447, 545-549. [CrossRef] [PubMed]

27. Zhao, Y.; Yang, R.; Rui, Q.; Wang, D. Intestinal Insulin Signaling Encodes Two Different Molecular Mechanisms for the Shortened Longevity Induced by Graphene Oxide in Caenorhabditis elegans. Sci. Rep. 2016, 6, 24024. [CrossRef] [PubMed]

28. Kwon, E.S.; Narasimhan, S.D.; Yen, K.; Tissenbaum, H.A. A new DAF-16 isoform regulates longevity. Nature 2010, 466, 498-502. [CrossRef] [PubMed]

29. Watts, J.L. Fat synthesis and adiposity regulation in Caenorhabditis elegans. Trends Endocrinol. Metab. 2009, 20, 58-65. [CrossRef] [PubMed] 
30. Rodriguez, M.; Snoek, L.B.; De Bono, M.; Kammenga, J.E. Worms under stress: C. elegans stress response and its relevance to complex human disease and aging. Trends Genet. 2013, 29, 367-374. [CrossRef] [PubMed]

31. Meng, F.; Li, J.; Wang, W.; Fu, Y. Gengnianchun, a Traditional Chinese Medicine, Enhances Oxidative Stress Resistance and Lifespan in Caenorhabditis elegans by Modulating daf-16/FoxO. Evid. Based Complement. Altern. Med. 2017. [CrossRef] [PubMed]

32. Mukhopadhyay, A.; Oh, S.W.; Tissenbaum, H.A. Worming pathways to and from DAF-16/FoxO. Exp. Gerontol. 2006, 41, 928-934. [CrossRef] [PubMed]

33. Tullet, J.M.A.; Green, J.W.; Au, C.; Benedetto, A.; Thompson, M.A.; Clark, E.; Gilliat, A.F.; Young, A.; Schmeisser, K.; Gems, D. The SKN-1/Nrf2 transcription factor can protect against oxidative stress and increase lifespan in C. elegans by distinct mechanisms. Aging Cell 2017, 16, 1191-1194. [CrossRef] [PubMed]

34. Seo, H.W.; Cheon, S.M.; Lee, M.Y.; Kim, H.J.; Jeon, H.; Cha, D.S. Catalpol Modulates Lifespan via DAF-16/FoxO and SKN-1/Nrf2 Activation in Caenorhabditis elegans. Evid. Based Complement. Altern. Med. 2015, 2015, 524878. [CrossRef] [PubMed]

35. Senchuk, M.M.; Dues, D.J.; Schaar, C.E.; Johnson, B.K.; Madaj, Z.B.; Bowman, M.J.; Winn, M.E.; Van Raamsdonk, J.M. Activation of $D A F-16 / F o x O$ by reactive oxygen species contributes to longevity in long-lived mitochondrial mutants in Caenorhabditis elegans. PLoS Genet. 2018. [CrossRef] [PubMed]

36. Murphy, C.T.; McCarroll, S.A.; Bargmann, C.I.; Fraser, A.; Kamath, R.S.; Ahringer, J.; Li, H.; Kenyon, C. Genes that act downstream of DAF-16 to influence the lifespan of Caenorhabditis elegans. Nat. Genet. 2003, 424, 277-283. [CrossRef] [PubMed]

37. Artyukhin, A.B.; Yim, J.J.; Cheong, M.; Avery, L. Starvation-induced collective behavior in C. elegans. Sci. Rep. 2015. [CrossRef] [PubMed]

38. Gengyo-Ando, K.; Mitani, S. Characterization of mutations induced by ethyl methanesulfonate, UV, and trimethylpsoralen in the nematode Caenorhabditis elegans. Biochem. Biophys. Res. Commun. 2000, 269, 64-69. [CrossRef] [PubMed]

39. Friedland, A.E.; Tzur, Y.B.; Esvelt, K.M.; Colaiácovo, M.P.; Church, G.M.; Calarco, J.A. Heritable genome editing in C. elegans via a CRISPR-Cas9 system. Nat. Methods 2013, 10, 741-743. [CrossRef] [PubMed]

40. Stiernagle, T. Maintenance of C. elegans. WormBook 2006, 1-11. [CrossRef] [PubMed]

41. Thomas, J.; Lea, K.; Zucker-Aprison, E.; Blumenthal, T. The spliceosomal snRNAs of Caenorhabditis elegans. Nucleic Acids Res. 1999, 18, 2633-2642. [CrossRef]

42. Kadandale, P.; Chatterjee, I.; Singson, A. Germline transformation of Caenorhabditis elegans by injection. Methods Mol. Biol. 2009, 518, 123-133. [PubMed]

43. Meyer, L.R.; Zweig, A.S.; Hinrichs, A.S.; Karolchik, D.; Kuhn, R.M.; Wong, M.; Sloan, C.A.; Rosenbloom, K.R.; Roe, G.; Rhead, B.; et al. The UCSC Genome Browser database: Extensions and updates 2013. Nucleic Acids Res. 2013, 41, D64-D69. [CrossRef] [PubMed]

44. Lewis, J.A.; Fleming, J.T. Basic Culture Methods. Methods Cell Biol. 1995, 48, 3-29. [PubMed]

45. Won, S.M.; Cha, H.U.; Yi, S.S.; Kim, S.J.; Park, S.K. Tenebrio molitor Extracts Modulate the Response to Environmental Stressors and Extend Lifespan in Caenorhabditis elegans. J. Med. Food 2016. [CrossRef] [PubMed]

46. Lee, E.B.; Xing, M.M.; Kim, D.K. Lifespan-extending and stress resistance properties of brazilin from Caesalpinia sappan in Caenorhabditis elegans. Arch. Pharm. Res. 2017, 40, 825-835. [CrossRef] [PubMed]

47. Possik, E.; Pause, A. Measuring oxidative stress resistance of Caenorhabditis elegans in 96-well microtiter plates. J. Vis. Exp. 2015, e52746. [CrossRef] [PubMed]

48. Walker, G.A.; Lithgow, G.J. Lifespan extension in C. elegans by a molecular chaperone dependent upon insulin-like signals. Aging Cell 2003, 2, 131-139. [CrossRef] [PubMed]

49. Rea, S.L.; Wu, D.; Cypser, J.R.; Vaupel, J.W.; Johnson, T.E. A stress-sensitive reporter predicts longevity in isogenic populations of Caenorhabditis elegans. Nat. Genet. 2005, 37, 894-898. [CrossRef] [PubMed]

(C) 2018 by the authors. Licensee MDPI, Basel, Switzerland. This article is an open access article distributed under the terms and conditions of the Creative Commons Attribution (CC BY) license (http:/ / creativecommons.org/licenses/by/4.0/). 